\section{Local and Systemic Immune Responses to Intratracheal Instillation of Antigen and DNA Vaccines in Mice}

\author{
Catherine Lombry, ${ }^{1}$ Aline Marteleur, ${ }^{1}$ \\ Mohammed Arras, ${ }^{2}$ Dominique Lison, ${ }^{2}$ \\ Jamila Louahed, ${ }^{3}$ Jean-Christophe Renauld, $^{3}$ \\ Véronique Préat, ${ }^{1}$ and Rita Vanbever ${ }^{1,4}$
}

Received April 28, 2003; accepted September 29, 2003

\begin{abstract}
Purpose. The purpose of this study was to determine the immunization efficacy of antigen and DNA vaccines after delivery to the lung, to assess the integrity of the pulmonary tissue after vaccination, and to elucidate mechanisms involved in the induction of immunity.

Methods. Ovalbumin, the plasmid encoding ovalbumin, the hepatitis B surface antigen ( $\mathrm{HBsAg}$ ), or plasmid encoding $\mathrm{HBsAg}$ were intratracheally instilled or injected in quadriceps in mice. The immune response and its Th polarization were analyzed over time. Markers of inflammation were measured in bronchoalveolar lavage, and lung histology was performed. The fate of ovalbumin following intratracheal instillation was studied.

Results. According to the vaccine, the pulmonary route produced stronger or equivalent humoral and cellular responses systemically and locally in the lung as compared to injection. The IgG subclasses and cytokine pattern indicate that the immunity was preferentially polarized toward the Th2 and Th1 type for antigen and DNA immunization, respectively. Ovalbumin penetrated the respiratory tissue and blood poorly after intratracheal instillation, suggesting that the immune response was triggered at airway surfaces. Overall, vaccines delivered to the lung did not induce any local sign of inflammation. Conclusions. Pulmonary administration of vaccines might be a promising alternative to conventional vaccination by injection.
\end{abstract}

KEY WORDS: antigens and DNA vaccines; fate of vaccine molecule; IgG; intratracheal instillation; lungs, proliferation assay.

\section{INTRODUCTION}

Because the majority of human pathogens initially infect through mucosal tissues, the nasal, pulmonary, or oral delivery of vaccines may facilitate the prevention of certain infections by inducing a mucosal immunity. Mucosal vaccines also induce a systemic immunity, which is essential to avoid

\footnotetext{
${ }^{1}$ Department of Pharmaceutical Technology, School of Pharmacy, Université catholique de Louvain (UCL), Brussels, Belgium.

${ }^{2}$ Industrial Toxicology and Occupational Medicine Unit, School of Medicine, UCL, Brussels, Belgium.

${ }^{3}$ Ludwig Institute for Cancer Research and Experimental Medicine Unit, UCL, Brussels, Belgium.

${ }^{4}$ To whom correspondence should be addressed. (e-mail: vanbever@ farg.ucl.ac.be)

ABBREVIATIONS: AUC, area under the curve; BAL, bronchoalveolar lavage; ELISA, enzyme-linked immunosorbent assay; HBsAg, hepatitis B surface antigen; HBSS, Hank's balanced salt solution; IFN- $\gamma$, interferon gamma; IgG, immunoglobulin G; IL-4, interleukin 4; i.m., intramuscular; i.t., intratracheal; i.v., intravenous; LDH, lactate dehydrogenase; ova, ovalbumin; pHBsAg, plasmid encoding hepatitis B surface antigen; pOVA, plasmid encoding ovalbumin.
}

spreading of the infection in the body. Delivery systems to mucosa are adaptable to mass campaigns and avoid the risks associated with injections. Mucosal immunization could therefore help to eradicate infectious diseases $(1,2)$.

Pulmonary administration of vaccines appears particularly promising because, in contrast to other mucosal surfaces, the lung contains a highly responsive immune system (3-7). Efficacious and safe immunization by inhalation of vaccines has been demonstrated in a few cases over the past 40 years. Several investigators reported that inhaled attenuated measles virus vaccine produced higher seroconversion rates, higher antibody titers, better antibody persistence, increased protection, and less clinical side effects such as fever, rash, rhinitis, cough, and headache in infants and children than vaccination by the subcutaneous route or by nasal instillation $(3,4,7)$. Preliminary human trials of inhaled inactivated influenza virus vaccine achieved identical outcomes (5).

Although pulmonary delivery of measles and influenza vaccines appeared highly effective and harmless, inhaled vaccines have remained poorly studied, and no approved vaccine aerosol is on the market yet. The physiological parameters responsible for the high efficacy are not understood, and there are no studies that have examined the integrity of the lung tissue after immunization as well as having analyzed the immunity produced by DNA vaccines after administration to the lung. In this article, we determined the overall immunization efficacy and safety of pulmonary antigen and naked DNA vaccines using ovalbumin and the hepatitis B surface antigen as model antigens. The immune responses, their $\mathrm{Th}$ polarization, and the alterations to the lung tissue were systematically assessed following intratracheal instillation of ovalbumin, hepatitis B surface antigen, or the plasmids encoding these antigens in mice. The fate of ovalbumin following intratracheal instillation was studied as a way to increase our understanding of the mechanisms involved in immunization.

\section{MATERIALS AND METHODS}

\section{Chemicals}

Ovalbumin (albumin from chicken egg white, 99\% albu$\mathrm{min}$ ) and xylazine were obtained from Sigma (Bornem, Belgium). The plasmid encoding ovalbumin (pOVA), hepatitis B surface antigen ( $\mathrm{HBsAg}$ ), and the plasmid encoding HBsAg (pHBsAg) were kindly donated by K. Thielemans (Hematology and Oncology Laboratory, Vrije Universiteit Brussel, Belgium), GlaxoSmithKline (Rixensart, Belgium) and Aldevron (Fargo, ND, USA), respectively. The construct pOVA was derived from the pcDNA3.1 expression vector containing a cytomegalovirus (CMV) promoter. pHBsAg [also named $\mathrm{pRc} / \mathrm{CMV}-\mathrm{HBs}(\mathrm{S})]$ expresses the hepatitis B surface antigen (small, or S, protein) under the control of the CMV immediate-early promoter. Milligram quantities of pOVA or pHBsAg were produced in Escherichia coli and purified using endofree plasmid mega kit from Qiagen (Hilden, Germany). The ovalbumin and pOVA preparations contained $2.5 \mathrm{ng}$ and $10 \mathrm{pg}$ of endotoxin per mg, respectively, as determined by the limulus amoebocyte lysate assay (Pyrogent Plus 64 Test Kit, $0.25 \mathrm{EU} / \mathrm{ml}$ sensitivity; Biowhittaker Europe, Verviers, Belgium). Imject Alum was obtained from Perbio (ErembogemAalst, Belgium). Ketamine (Ketalar) was purchased from 
Warner-Lambert (Zaventem, Belgium) and natrium pentobarbital from Certa (Medeva Pharma, Braine l'Alleud, Belgium). Sterile $\mathrm{NaCl} 0.9 \%$ solutions were supplied by Braun Medical N.V./S.A. (Diegem, Belgium) and Hank's balanced salt solution (HBSS) without $\mathrm{Ca}^{2+}, \mathrm{Mg}^{2+}$, and phenol-red by Life Technologies (Merelbeke, Belgium).

\section{Mice}

Specific pathogen-free female NMRI mice aged 6 weeks were purchased from Elevage Janvier (Le Genest St. Isle, France). The mice were kept in hanging wire cages and allowed to feed and drink ad libitum.

\section{Immunizations}

Mice were anesthetized using ketamine/xylazine (90/10 $\mathrm{mg} / \mathrm{kg}$ ) intraperitoneal injection before immunization. For intratracheal instillation, the trachea was exposed; a small incision between two cartilaginous rings was made using a $23 \mathrm{G}$ sterile needle (Terumo, Leuven, Belgium), and a metal tube of smaller diameter $(26 \mathrm{G})$ was loosely inserted in the aperture. $\mathrm{NaCl} 0.9 \%$ solutions of ovalbumin $(100 \mu \mathrm{g}$ in $30 \mu \mathrm{l})$, pOVA $(100 \mu \mathrm{g}$ in $30 \mu \mathrm{l})$, and pHBsAg $(100 \mu \mathrm{g}$ in $25 \mu \mathrm{l})$ or $\mathrm{NaCl} 0.9 \%$ suspension of $\mathrm{HBsAg}(10 \mu \mathrm{g}$ in $25 \mu \mathrm{l})$ were instilled through the tube. Immediately after instillation, five $150-\mu l$ air bolus were successively insufflated in the lung using a 1-ml standard syringe connected to the endotracheal tube. The air volume of $150 \mu \mathrm{l}$ was chosen because it corresponds to the tidal volume in mice (8). Spreading of the instillate throughout the lung was ascertained by visual inspection of the lung following delivery of Tryptan blue (data not shown). The mice were sutured and allowed to recover. For intramuscular dosing, $100 \mu \mathrm{g}$ of ovalbumin, pOVA, and pHBsAg or $10 \mu \mathrm{g}$ of $\mathrm{HBsAg}$ were dissolved or suspended in $100 \mu \mathrm{l}$ of saline or $10 \mu \mathrm{g}$ of HBsAg was suspended in $100 \mu \mathrm{l}$ of saline/ Imject Alum 50/50, and the solutions or suspensions were injected in quadriceps (50 $\mu \mathrm{l}$ in each quadriceps) (9-11). For intranasal administration, $10 \mu \mathrm{g}$ of $\mathrm{HBsAg}$ or $100 \mu \mathrm{g}$ of pHBsAg in $10 \mu \mathrm{l}$ of $\mathrm{NaCl} 0.9 \%$ were deposited in the mouse nostrils $(5 \mu \mathrm{l} /$ nostril; small volumes allow to hold the solution in the upper respiratory tract (9)) under light ether anesthesia. No mice died during any of the immunization procedures, including the intratracheal instillation procedure. All experimental protocols in mice were approved by the Institutional Animal Care and Use Committee of the Université catholique de Louvain.

\section{Measurement of Antibody Responses}

Mice were immunized intratracheally (i.t.) or intramuscularly (i.m.) with ovalbumin, pOVA, HBsAg, or pHBsAg either on days 0,14 , and 28 or on days 0 and 28 , as described above. Sera were collected by orbital bleeding before each immunization and then at 2-week intervals up to week 10 . A few mice in each group died during blood sampling. At week 10 postinitial administration, mice were killed with an overdose of pentobarbital, and bronchoalveolar lavages (BALs) were performed. $1 \mathrm{ml}$ of HBSS was slowly injected in the trachea, left in the lung for $15 \mathrm{~s}$, withdrawn, re-instilled, and withdrawn two additional times. After centrifugation $(1000 \mathrm{~g}$ for $10 \mathrm{~min}$ at $4{ }^{\circ} \mathrm{C}$ ), the resultant cell-free supernatant was analyzed for $\mathrm{IgG}$ titers, as was the serum.
Levels of anti-ovalbumin or anti-HBsAg antibodies were determined by enzyme-linked immunosorbent assay (ELISA) in serum and BAL from individual mice (5 to 10 mice per group). Serial dilutions of sera or BAL were made in ovalbumin- or HBsAg-coated plates (Nunc-Immuno Plate Maxisorp Surface, Gibco BRL Life Technologies, Mgrelbeke, Belgium), and detection of anti-ovalbumin or anti-HBsAg antibodies was carried out using peroxidase-labeled rat antimouse immunoglobulin $\mathrm{G}$ (total $\mathrm{IgG}$ ), IgG1, IgG2a, IgG2b, IgA, and IgE (LO-IMEX, Brussels, Belgium). Titers were defined as the highest dilution to give an optical density of 0.2 at $450 \mathrm{~nm}$. One hundred percent of the mice responded in the groups immunized with ovalbumin (i.t. and i.m.), HbsAg (i.t. and i.m.) and pHBsAg (i.t.). In the groups immunized with pOVA (i.t. and i.m.), 60-80\% were responders. Zero titers were arbitrarily given to nonresponders and included in mean titers (Figs. 1 and 2). No anti-ovalbumin or anti-HBsAg antibodies were detected in mice that had not been vaccinated with ovalbumin, pOVA, HBsAg, or pHBsAg (data not shown)

The avidity of the anti-ovalbumin antibodies was determined by competition ELISA, in which increasing concentrations of free antigen are added to the wells to inhibit competitively the binding of antibodies of decreasing affinities (12). $0.0,0.1,0.5,1.0$, and $5.0 \mu \mathrm{g} / \mathrm{ml}$ dilutions of soluble ovalbumin were added to the serial dilutions of sera. The percentage of inhibition was plotted against the concentration of inhibitor, and the concentration of inhibitor required for $50 \%$ inhibition was taken as a measure of avidity. The assessment of antibody avidities was performed on pooled sera collected 8 weeks postinitial vaccination in each group of mice.

\section{Cytokine Detection and Proliferation Assay}

Mice were immunized i.t. or i.m. with $100 \mu \mathrm{g}$ of ovalbumin or pOVA on days 0,14 , and 28 , as described above. Ten days after the last immunization, the mice were boosted with $10 \mu \mathrm{g}$ of soluble ovalbumin by the same route of administration as the initial immunization. Three days postboosting, mice were sacrificed, and spleens as well as parathymic and posterior-mediastinal lymph nodes removed aseptically. Spleens and lymph nodes from four to six mice were analyzed individually in each group; a nonimmunized control group was also included.

For cytokine assays, splenocytes were adjusted to a concentration of $5 \times 10^{6}$ cells $/ \mathrm{ml}$ and cultured in flat microwell plates (Nunclon; catalog no. 167008; Nunc, Wiesbaden, Germany) in DMEM medium supplemented with $0.5 \%$ NMRI mice serum, $50 \mathrm{mM}$ 2-mercaptoethanol, $0.55 \mathrm{mM}$ L-arginine, $0.24 \mathrm{mM}$ L-asparagine, $1.25 \mathrm{mM} \mathrm{L}$-glutamine, and $200 \mu \mathrm{g} / \mathrm{ml}$ ovalbumin. Cells were incubated at $37^{\circ} \mathrm{C}$ in a humidified $\mathrm{CO}_{2}$ incubator, and supernatants were harvested after $48 \mathrm{~h}$ for interferon-gamma (IFN- $\gamma$ ) and interleukin-4 (IL-4) assays (Mouse IFN- $\gamma$ and IL-4 DuoSet ELISA Development kits, R\&D Systems Europe Ltd, Abingdon, UK). The limits of detection of the IFN- $\gamma$ and IL-4 assays were 31 and $63 \mathrm{pg} / \mathrm{ml}$, respectively. Sixty to $70 \%$ of the mice responded in the assay following immunization with pOVA, and 33\% responded following immunization with ovalbumin. Only the data from the groups with $>60 \%$ responders were mentioned. No cytokines were detected when naive control splenocytes were stimulated by ovalbumin.

For proliferation assays, splenocytes and lymphocytes 
collected from lung-draining lymph nodes were adjusted to a concentration of $10^{6}$ and $5 \times 10^{5}$ cells $/ \mathrm{ml}$, respectively, in DMEM medium enriched as above and supplemented with ovalbumin (final concentration, $200 \mu \mathrm{g} / \mathrm{ml}$ ) or concanavalinA, a T-cell mitogen (final concentration, $5 \mu \mathrm{g} / \mathrm{ml}$ ) or neither of them. The cells were incubated for $48 \mathrm{~h}$ and then pulsed with $0.5 \mu \mathrm{Ci}\left[{ }^{3} \mathrm{H}\right]$ thymidine per well overnight. Concanavalin-A induced the proliferation of splenocytes in all cases, indicating that the cells were fully functional after cell isolation and plating.

\section{Fate of Ovalbumin following Intratracheal Instillation}

One hundred micrograms of ovalbumin was delivered by i.t. instillation or i.m. injection as described above or by intravenous (i.v.) injection in the ophthalmic venous plexus. Blood samples $(150 \mu \mathrm{l})$ were periodically drawn by orbital puncture over a period of $24 \mathrm{~h}$ and the sera stored at $-20^{\circ} \mathrm{C}$ until analysis. No more than three blood samples were withdrawn per mouse, and the entire pharmacokinetics was therefore collected by distributing sampling times among two (i.t. and i.m.) or three (i.v.) groups of mice. Ovalbumin concentrations in serum were measured by ELISA using nonconjugated and horse radish peroxidase-labeled rabbit antiovalbumin antibodies (Research Diagnostics Inc., Flanders, NJ, USA). The detection limit of the assay was $0.5 \mathrm{ng} / \mathrm{ml}$. The areas under the serum ovalbumin curves (AUC) were calculated on average data using the trapezoidal rule. The absolute bioavalability values of the i.t. and i.m. administrations were calculated by comparison to i.v. as $\mathrm{AUC}_{\text {i.t. }}$ or i.m. $/ \mathrm{AUC}_{\mathrm{i.v}}$. $\times 100$. Experiments were performed using 12 to 14 mice for each route of administration, and serum ovalbumin concentrations at each sampling time were the average from five to six mice.

Ovalbumin was also quantified in the air space, lung tissue, and/or serum 0,4 , and $24 \mathrm{~h}$ following i.t. and i.m. deliveries of $100 \mu \mathrm{g}$ of the antigen or pOVA. Ovalbumin quantification in the air space and tissue was performed in BAL (5 times $1 \mathrm{~mL}$ of HBSS) and excised minced lungs, respectively. The lung lobes were minced in ultrapure water for 2 min using a Potter tissue grinder (Merck Eurolab, Leuven, Belgium); the tissue homogenate was then centrifuged at $8000 \mathrm{~g}$ for $15 \mathrm{~min}$ and the supernatant kept for ovalbumin assay. The quantities of ovalbumin remaining in the metal tube used for i.t. delivery were also measured. When known ovalbumin quantities were added to lung tissue, BAL, or serum, they were entirely recovered by ELISA, indicating that degradation or tissue binding was insignificant during sample preparation. The amount of ovalbumin recovered in each sample was expressed as percentage of the dose delivered. Serum volume was estimated at $10 \%(\mathrm{v} / \mathrm{w})$ of body weight. Data at each sampling time are the averages from three mice.

\section{Analysis of Inflammation Markers in Bronchoalveolar Lavage and Lung Histology}

Mice were immunized i.t. with $100 \mu \mathrm{g}$ of ovalbumin or pOVA either on day 0 or on days 0 and 28. BALs ( 3 times 1 $\mathrm{ml}$ ) were performed as described above $4 \mathrm{~h}, 24 \mathrm{~h}$, or 5 days following the priming or boost dose. The first cell-free supernatant was used for determination of total protein content and lactate dehydrogenase $(\mathrm{LDH})$ activity. Total protein as- say is a measure of damage to the alveolar capillary barrier that hinders transudation of serum proteins. LDH is an intracellular enzyme and its activity in BAL measures cell damage or lysis (13). Protein concentrations were determined by the Pyrogallol Red staining method using human albumin as standard (Technicon RA Systems, Bayer Diagnostics, Domont, France). LDH activity was measured spectrophotometrically by monitoring the reduction of $\mathrm{NAD}^{+}$at $340 \mathrm{~nm}$ in the presence of lactate (Technicon RA Systems). The cell pellets were resuspended in $300 \mu \mathrm{l}$ of sterile HBSS, and the total number of leukocytes was determined by Türch staining. The cell differential counting was performed on cytocentrifuge preparations fixed in methanol and stained with Diff Quick (Dade Berhing AG, Düdingen, Switzerland; 300 cells per mouse) (13). Each group included three to eight mice.

In another set of experiments, the lungs were removed $24 \mathrm{~h}$ following i.t. instillation of a priming or boost dose of ovalbumin or pOVA and placed in a $4 \%$ formol solution. A control group was i.t. instilled with saline and another one left untreated. Paraffin-embedded slices were stained with hematoxylin-eosin for histopathological examination by light microscopy.

\section{Statistical Analysis}

The data presented in Figs. 1, 2, and 5 were analyzed by Anova or $t$ test $(\mathrm{p}<0.05)$, the data in Fig. 3 by Pearson correlation, and the data in Fig. 4 and in Table I by KruskalWallis test $(\mathrm{p}<0.05$ or 0.01$)$ because variances were not equal among groups ( $\mathrm{p}<0.05$, Levene's test).

\section{RESULTS}

\section{Antibody Responses to Intratracheal Instillation of Vaccines}

Ovalbumin or pOVA were delivered to the lung by i.t. instillation or injected in both quadriceps of NMRI mice at a dose of $100 \mu \mathrm{g}$ on two or three occasions. The anti-ovalbumin antibody titers then generated in serum and locally in the lung were quantified over time. Ovalbumin induced stronger antibody responses in serum and in the lung when delivered by i.t. instillation than by i.m. injection (Fig. $1 \mathrm{a}$ and b; p < 0.05). The plasmid induced 2 logs lower IgG titers than the antigen and levels that were equivalent by both routes (Fig. 1a; $\mathrm{p}>0.05)$. The only isotype produced was IgG; no IgE or IgA were detected in serum and BAL (data not shown).

The functional avidities of the anti-ovalbumin immunoglobulins were identical among groups, as assessed by competition ELISA (12). The concentration of ovalbumin solution added to the wells which resulted in a $50 \%$ binding inhibition of each sera control was approximately $3.5 \mu \mathrm{g} / \mathrm{ml}$ for the four groups of mice.

Decreasing the antigen dose from 100 to $10 \mu \mathrm{g}$ decreased total anti-ovalbumin IgG titers by 2 logs in serum and $0.3-0.4$ $\log$ in BAL, both following administration by i.t. instillation as well as i.m. injection (data not shown). This indicates that the level of immunization could be controlled by the dose.

In order to assess the effectiveness of mucosal vaccination by the pulmonary route in general, we immunized mice with HBsAg or pHBsAg and analyzed the antibodies produced. Administration of HBsAg to the lung generated 

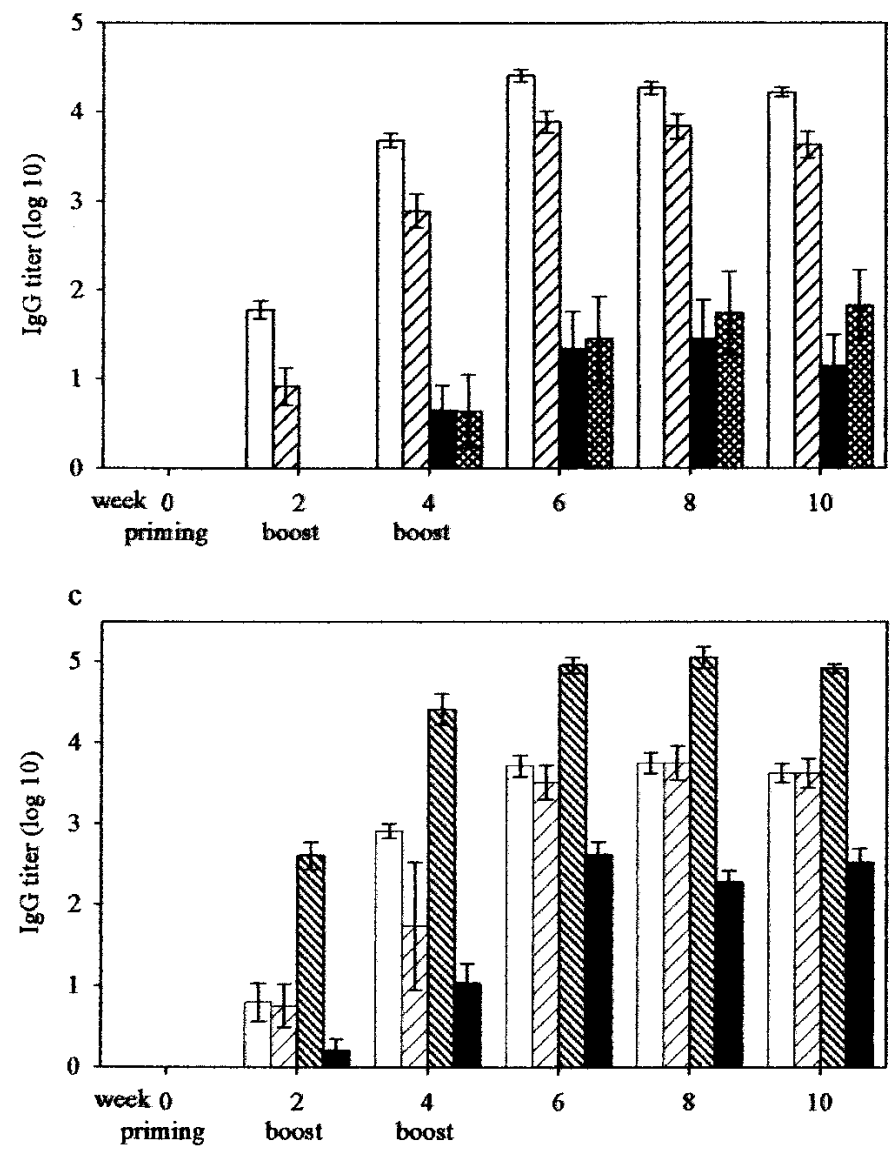

b
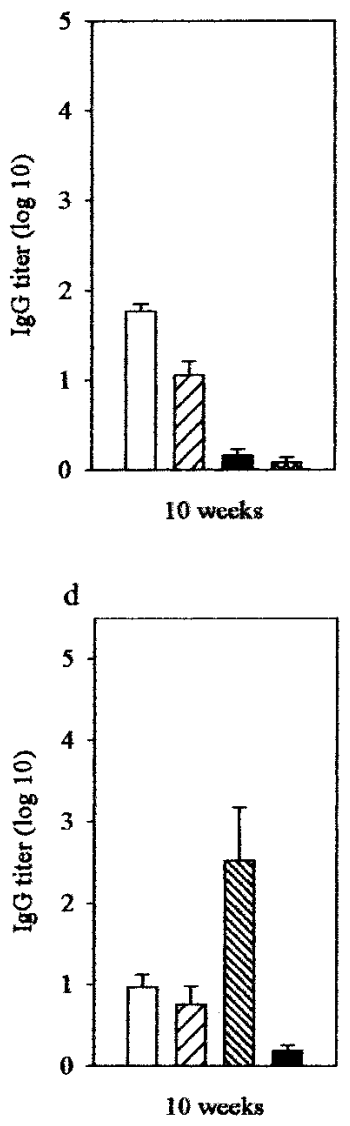

Fig. 1. Influence of the route of administration of antigen and DNA vaccines on the generation of specific total $\operatorname{IgG}(\mathrm{a}, \mathrm{c})$ in serum and $(\mathrm{b}, \mathrm{d})$ in BAL. $(\mathrm{a}, \mathrm{b})$ One hundred micrograms of ova or pOVA were i.t. instilled into the lung or injected i.m. on three occasions in NMRI mice. (c, d) In the same manner, $10 \mu \mathrm{g}$ of HBsAg or $100 \mu \mathrm{g}$ of pHBsAg were delivered to the lungs, nostrils, or muscles with or without alum. (b, d) Ten weeks postinitial immunization, the mice were sacrificed and the lung

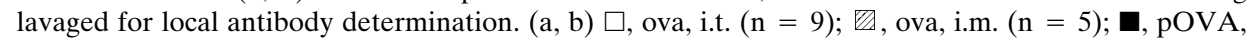

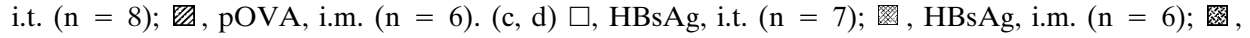
HBsAg with alum, i.m. $(\mathrm{n}=6)$; $\boldsymbol{\square}$, pHBsAg, i.t. $(\mathrm{n}=5)$. No specific antibodies were detected after intranasal administration of HBsAg or pHBsAg and after i.m. injection of pHBsAg, and null titers are not shown for clarity. Results are expressed as the mean of the $\log 10$ titer \pm SEM.

equivalent total anti-hepatitis B IgG titers in serum and BAL as its i.m. injection ( $\mathrm{p}>0.05)$. In comparison, $\mathrm{IgG}$ titers were one order of magnitude higher after i.m. injection of $\mathrm{HBsAg}$ formulated with alum $(\mathrm{p}<0.05)$ and one order of magnitude lower after i.t. instillation of pHBsAg (Figs. $1 \mathrm{c}$ and d; $\mathrm{p}<0.05)$. No specific antibodies were detected after i.m. injection of pHBsAg. Administration of HBsAg or pHBsAg to the mouse nostrils was also performed, but nasal vaccination did not yield any detectable specific immunity in our conditions (data not shown). As with ovalbumin, HBsAg and pHBsAg generated only the isotype $\operatorname{IgG}$ and no $\operatorname{IgE}$ or $\operatorname{IgA}$.

\section{Type of Immune Response}

The analysis of IgG subclasses and the quantification of cytokines secreted by splenocytes in culture indicated that the immune response was Th2-polarized after i.t. instillation and i.m. injection of ovalbumin, mixed Th1/Th2 after i.t. instillation of pOVA, and Th1-polarized after i.m. injection of pOVA. The antibodies in serum were IgG1-dominated fol- lowing i.t. instillation of ovalbumin and pOVA as well as following i.m. injection of ovalbumin, indicating a Th2 component (Fig. 2a). The injection of the plasmid in the muscle produced a mixed $\mathrm{IgG} 1, \mathrm{IgG} 2 \mathrm{a}$, and $\mathrm{IgG} 2 \mathrm{~b}$ response in serum, suggesting a Th1 component. The only IgG subclass detected in BAL was IgG1; no IgG2a or IgG2b could be measured in the four groups of mice (data not shown). Splenocytes cultured in vitro with ovalbumin produced the Th1 cytokine, interferon-gamma (IFN- $\gamma$ ), in the case of both routes of DNA immunization (data not shown).

The profile of the IgG subclasses produced after i.t. instillation or i.m. injection of HBsAg was dominated by $\mathrm{IgG} 1$ as well; however, a significant presence of $\operatorname{IgG} 2 \mathrm{a}$ and $\mathrm{IgG} 2 \mathrm{~b}$ was observed (Fig. 2b). HBsAg forms by self-assembly of 100 polypeptide monomers of 24-kDa into 22-nm lipoprotein particles; the physical nature and composition of HBsAg might cause a shift of the immunity toward the Th1 type $(11,14,15)$. The mixed $\mathrm{IgG} 1, \mathrm{IgG} 2 \mathrm{a}$, and $\mathrm{IgG} 2 \mathrm{~b}$ response produced after i.t. instillation of pHBsAg is indicative of a Th1 immune response (Fig. 2b). 

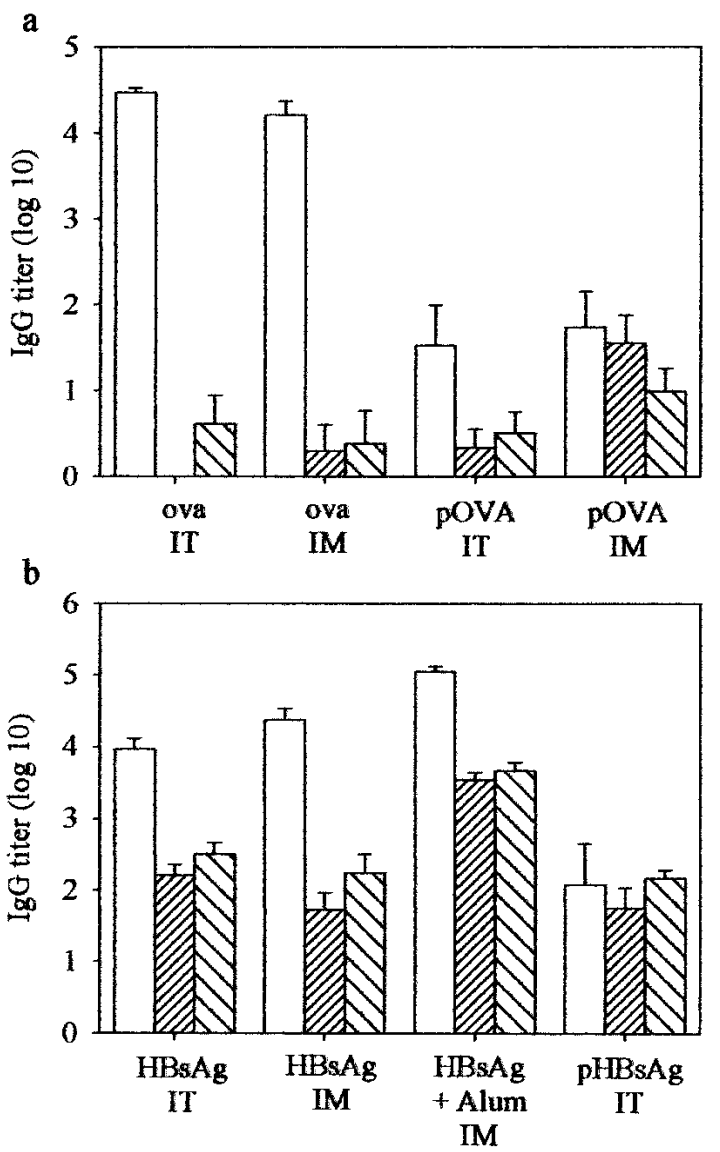

Fig. 2. IgG subclasses generated in serum 10 weeks postinitial immunization with (a) ova or pOVA and (b) HBsAg or pHBsAg by i.t. instillation or i.m. injection in NMRI mice. The experiment is the same as in Fig. 1. $\square$, IgG1; 罗, IgG2a; , IgG2b. The proportions of IgG subclasses were constant over the 10 weeks of the experiment (data not shown). Results are expressed as the mean of the log10 titer \pm SEM from five to nine mice in each group.

\section{Localization of the Immune Response}

It has been demonstrated that pulmonary dendritic cells are recruited into the airway epithelium within $1 \mathrm{~h}$ after i.t. injection of an antigen load, at which point they develop antigen-presenting-cell activities and migrate to regional lymph nodes over several days after contact with the antigen $(16,17)$. However, these studies did not assess the involvement of remote lymphoid organs such as the spleen in the immune response. Other authors have hypothesized that the high efficacy of inhaled vaccines could originate from efficient absorption of the antigen from the airway lumen to the bloodstream and processing of the absorbed antigen in specialized lymphoid organs in the body $(18,19)$. To get insights into the localization of the immune response produced by intratracheal instillation of vaccines, we examined the relationship between IgG levels in BAL and serum, we assessed the involvement of the lymphocytes from the spleen as well as lung draining lymph nodes in the immunization, and we studied the fate of ovalbumin following i.t. instillation.

The anti-ovalbumin IgG titers found locally in the lung 10 weeks postinitial immunization correlated with their serum counterparts following both i.t. and i.m. deliveries of the vaccines, and the regression lines through the i.t. and i.m. data a

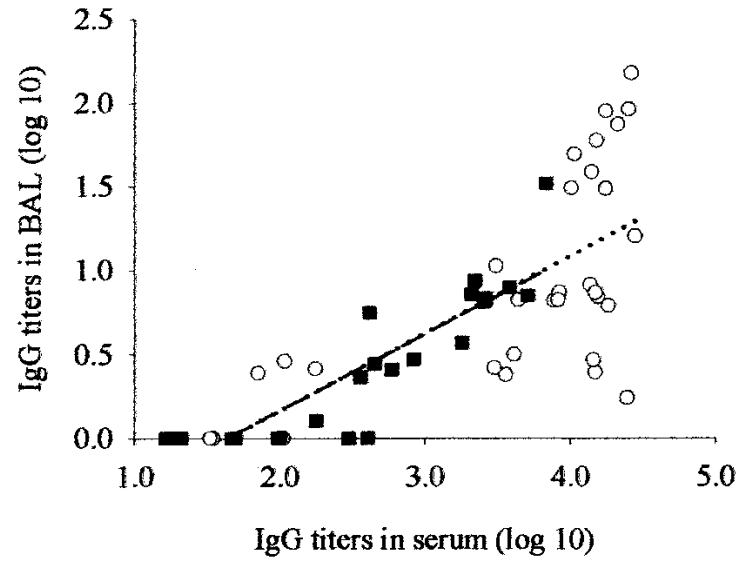

- IM: $y=0.46 x-0.74 r^{2}=0.77 p<0.01$

IT: $y=0.46 x-0.75 r^{2}=0.46 p<0.01$

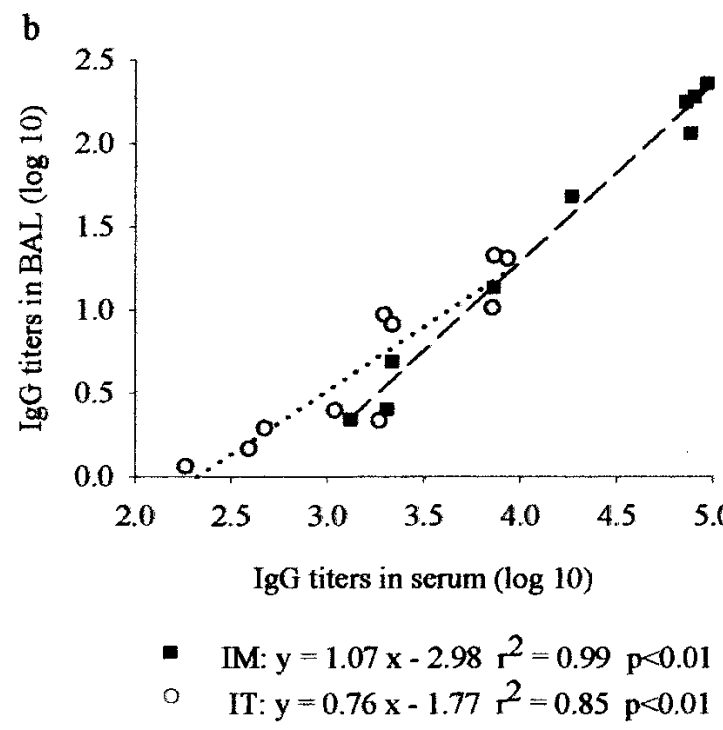

Fig. 3. Correlation between total $\mathrm{IgG}$ titers in BAL and in serum 10 weeks postinitial administration of (a) ova or pOVA and (b) HBsAg or pHBsAg to the lung (i.t.) or to quadriceps (i.m.) in NMRI mice. The experiment is the same as in Fig. 1. Results from individual mice are shown for both routes of administration. The correlations between titers are highly significant (Pearson test, $\mathrm{p}<0.01$ ).

were close to each other (Fig. 3a). Correlations between BAL and serum IgG titers were also found for antibodies produced against the hepatitis B surface antigen (Fig. 3b). This suggests a dependence of the immunoglobulin levels in the lung on those in serum. IgGs either diffused from blood to air spaces or B cells producing specific IgG were as abundant in the lung after i.m. than after i.t. delivery.

Splenocytes brought in contact with ovalbumin proliferated whether vaccination against ovalbumin had been carried out intratracheally or intramuscularly and whether the protein or the DNA had been delivered (Fig. 4a; $\mathrm{p}<0.05$ ). However, proliferation of splenocytes tended to be lower following i.m. injection of the protein as compared to the other groups $(p>0.05)$. In contrast, lymphocytes harvested from the parathymic and posterior-mediastinal lymph nodes proliferated only following delivery of ovalbumin or pOVA to 
a

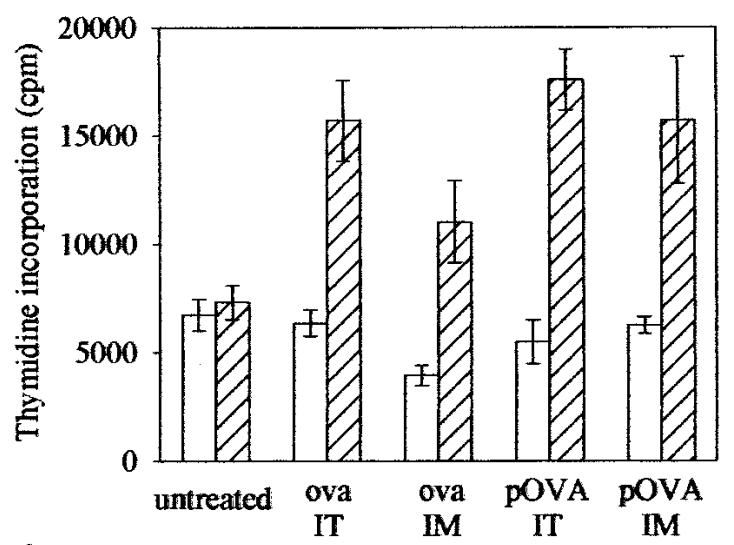

b

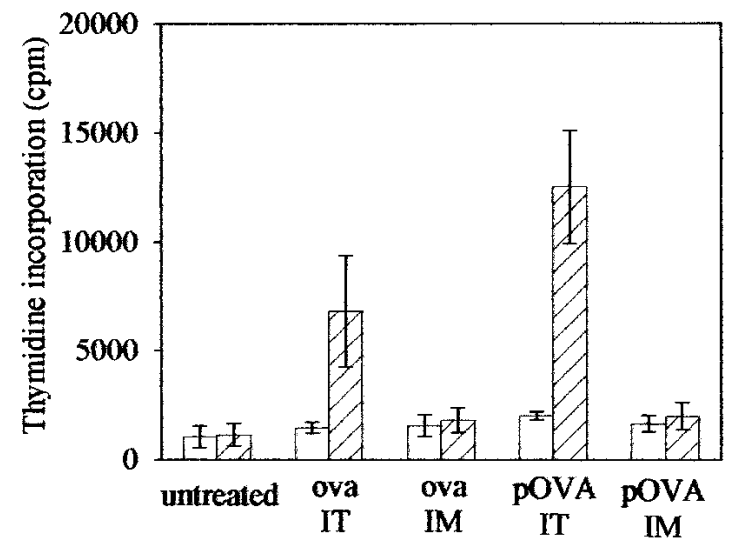

Fig. 4. Proliferation of (a) splenocytes and (b) lymphocytes harvested from lung draining lymph nodes following i.t. instillation of ova $(\mathrm{n}=5)$ or pOVA $(\mathrm{n}=6)$ or i.m. injection of ova $(\mathrm{n}=6)$ or pOVA $(\mathrm{n}=4)$ in NMRI mice. The control group $(\mathrm{n}=3)$ was not immunized in vivo. Spleens and lymph nodes were analyzed individually in each group and ( $\left.{ }^{2}\right)$ with or $(\square)$ without ovalbumin stimulation in vitro. The data are presented as mean \pm SEM for each group.

the lung $(\mathrm{p}<0.05)$, and a trend toward stronger proliferation was observed for the DNA vaccine as compared to the antigen (Fig. 4b; $\mathrm{p}>0.05$ ). This demonstrates that cellular immune mechanisms operated locally in the draining lymph nodes, but also systemically following vaccine delivery to the lung.

The absorption of ovalbumin in the systemic circulation following i.t. instillation and i.m. injection were both low, and lower following i.t. (absolute bioavailability of $14 \pm 3 \%$, SD) than i.m. delivery (absolute bioavailability of $25 \pm 5 \%$; $p<0.05$; Fig. 5a). Because the immunization efficacy of ovalbumin was higher following i.t. instillation than i.m. injection (Fig. 1a), it indicates that efficacy did not principally originate from efficient blood transport of antigen to the spleen for processing. The quantification of ovalbumin in the lung and serum of mice following i.t. instillation showed similar results; that is, a low tissue as well as systemic absorption and a prolonged presence of the macromolecule in the air space (Fig. 5b). The presence of antigen for hours in the airway lumen might favor surface immunization mechanisms, such as the stimulation of the dendritic cells present in the epithelial lining of the respiratory tract. No ovalbumin could be detected in the lung or serum following i.t. or i.m. delivery a
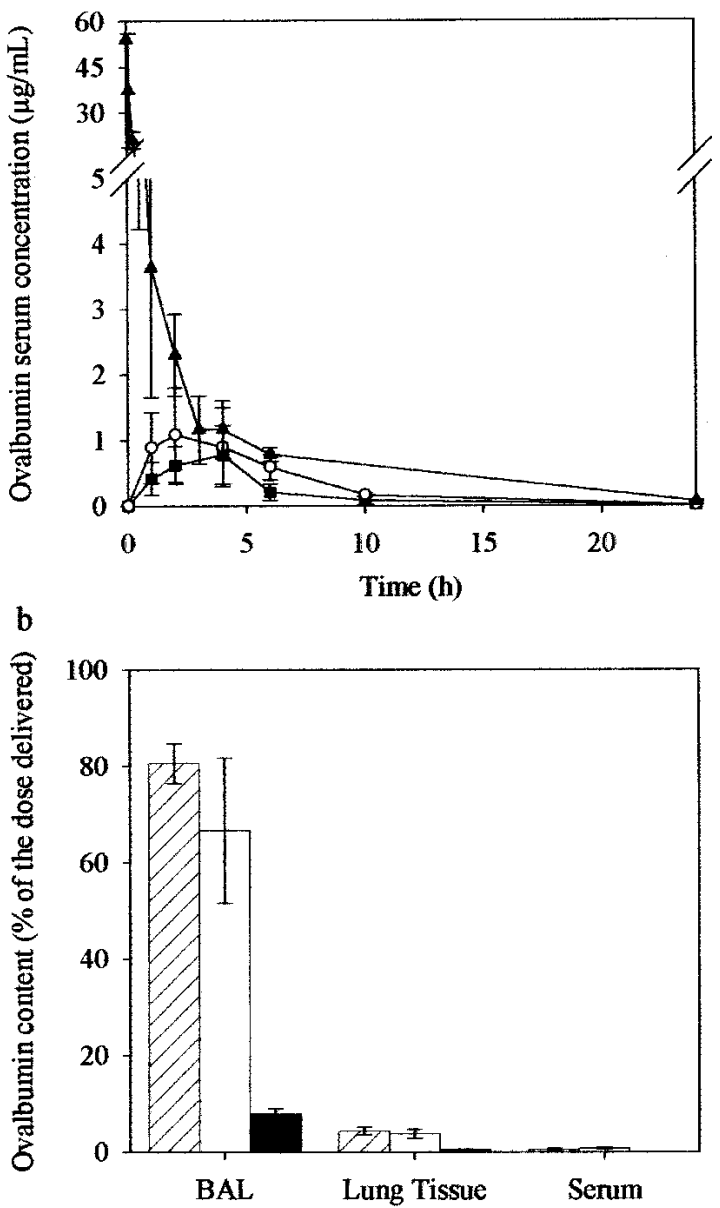

Fig. 5. Fate of ovalbumin following i.t. instillation in NMRI mice. (a) One hundred micrograms of ova were delivered by i.t. instillation ( $\mathbf{\square}$; $\mathrm{n}=6)$, i.m. $(\bigcirc ; \mathrm{n}=6)$, or i.v. $(\boldsymbol{\Delta} ; \mathrm{n}=5)$ injection, and serum concentrations were measured over time. Intratracheal instilled and i.m. injected ovalbumin were absorbed in the systemic circulation with an absolute bioavailability of $14 \pm 3 \%$ (SD) and $25 \pm 5 \%$, respectively. (b) One hundred micrograms of ova was i.t. instilled, and the fractions of the dose delivered recovered in BAL, lung tissue, and serum were determined $0 \mathrm{~h}(\mathbb{Q} ; \mathrm{n}=3), 4(\square ; \mathrm{n}=3)$ and $24 \mathrm{~h}(\boldsymbol{\square}$; $\mathrm{n}=3)$ after delivery. The data are presented as mean \pm SEM for each group.

of pOVA, indicating that ovalbumin concentrations in biological fluids produced by the plasmid were below the $\mathrm{ng} / \mathrm{ml}$ range.

\section{Safety Studies}

The mice intratracheally instilled with ovalbumin, HBsAg, pHBsAg, or pOVA in the above experiments did not show any external signs of toxicity, such as difficulty in breathing. Further assessment of the effects of vaccines on the lung was made by analysis of soluble and cellular components in BAL and histology of the lung. Measurements were made $4 \mathrm{~h}, 24 \mathrm{~h}$, and/or 5 days following i.t. instillation of a priming dose of ovalbumin or pOVA or following priming (week 0 ) and boost (week 4) doses of the vaccines. There was no increase in biochemical or cellular markers at any time after the i.t. instillation of ovalbumin or pOVA as compared to saline, except for a limited and transient increase in the percentage 
Table I. Toxicity Markers in Bronchoalveolar Lavage Following Immunization by the Intratracheal Route

\begin{tabular}{|c|c|c|c|c|c|c|c|}
\hline & \multirow[b]{2}{*}{ Group } & \multicolumn{3}{|c|}{ One instillation } & \multicolumn{3}{|c|}{ Two instillations } \\
\hline & & $4 \mathrm{~h}$ & $24 \mathrm{~h}$ & 5 days & $4 \mathrm{~h}$ & $24 \mathrm{~h}$ & 5 days \\
\hline \multirow[t]{4}{*}{ Total protein $(\mu \mathrm{g} / \mathrm{ml})$} & No treatment & $147 \pm 26$ & $120 \pm 10$ & $124 \pm 8$ & $96 \pm 9$ & $131 \pm 10$ & $113 \pm 31$ \\
\hline & Saline & $136 \pm 14$ & $123 \pm 11$ & $119 \pm 8$ & $143 \pm 12$ & $142 \pm 15$ & $97 \pm 6$ \\
\hline & ova & $141 \pm 3$ & $158 \pm 22$ & $106 \pm 3$ & $162 \pm 29$ & $104 \pm 17$ & $119 \pm 14$ \\
\hline & pOVA & $166 \pm 30$ & $144 \pm 17$ & $104 \pm 16$ & $125 \pm 22$ & $143 \pm 9$ & $104 \pm 6$ \\
\hline \multirow{4}{*}{ LDH activity (IU/l) } & No treatment & $72 \pm 12$ & $55 \pm 7$ & $46 \pm 2$ & $43 \pm 3$ & $53 \pm 3$ & $113 \pm 34$ \\
\hline & Saline & $59 \pm 5$ & $67 \pm 8$ & $50 \pm 1$ & $49 \pm 2$ & $68 \pm 10$ & $64 \pm 24$ \\
\hline & ova & $49 \pm 4$ & $79 \pm 8$ & $45 \pm 2$ & $118 \pm 47$ & $48 \pm 8$ & $67 \pm 16$ \\
\hline & pOVA & $67 \pm 7$ & $78 \pm 12$ & $39 \pm 3$ & $77 \pm 17$ & $61 \pm 5$ & $59 \pm 4$ \\
\hline \multirow[t]{4}{*}{ Number of white blood cells recovered $\left(\times 10^{4}\right)$} & No treatment & $8.6 \pm 2.1$ & $10.0 \pm 2.0$ & $7.1 \pm 2.5$ & $8.6 \pm 0.7$ & $12.7 \pm 2.3$ & $4.4 \pm 1.3$ \\
\hline & Saline & $14.9 \pm 4.2$ & $6.9 \pm 1.8$ & $9.8 \pm 2.6$ & $11.9 \pm 2.1$ & $9.1 \pm 3.0$ & $3.8 \pm 0.4$ \\
\hline & ova & $4.7 \pm 0.7$ & $7.5 \pm 0.9$ & $18.3 \pm 9.3$ & $12.6 \pm 3.8$ & $8.9 \pm 1.2$ & $6.5 \pm 0.7$ \\
\hline & pOVA & $13.1 \pm 2.4$ & $8.7 \pm 1.3$ & $5.1 \pm 1.4$ & $10.9 \pm 2.3$ & $5.6 \pm 0.6$ & $6.6 \pm 1.0$ \\
\hline \multirow[t]{4}{*}{ Percentage of neutrophils } & No treatment & $0.2 \pm 0.1$ & $0.1 \pm 0.1$ & $0.1 \pm 0.1$ & $0.1 \pm 0.1$ & $0.0 \pm 0.0$ & $0.0 \pm 0.0$ \\
\hline & Saline & $1.5 \pm 0.4$ & $0.6 \pm 0.3$ & $0.2 \pm 0.1$ & $7.7 \pm 3.5$ & $1.2 \pm 0.8$ & $0.0 \pm 0.0$ \\
\hline & ova & $11.4 \pm 5.4$ & $11.9 \pm 2.6^{*}$ & $0.3 \pm 0.3$ & $7.9 \pm 2.5$ & $7.0 \pm 1.0^{*}$ & $0.4 \pm 0.2$ \\
\hline & pOVA & $2.3 \pm 0.2$ & $2.0 \pm 0.5^{*}$ & $0.3 \pm 0.2$ & $7.9 \pm 2.9$ & $4.1 \pm 0.7^{*}$ & $0.3 \pm 0.1$ \\
\hline
\end{tabular}

One hundred micrograms of ovalbumin (ova) or plasmid encoding ovalbumin (pOVA) was intratracheally instilled in the lungs of NMRI mice either on day 0 or on days 0 and 28 . Four hours, 24 h, or 5 days following the priming or boost dose, bronchoalveolar lavage was performed and the total protein content, the activity of the lactate dehydrogenase (LDH), and the total and differential counts of white blood cells analyzed. Control groups were intratracheally instilled with saline or left untreated. Results are expressed as the mean \pm SEM from three to eight mice in each group. No increase in biochemical and cellular markers was noted at any time after the instillation of ovalbumin or pOVA as compared to saline, except a slight and transient increase in the percentage of neutrophils at $24 \mathrm{~h} .{ }^{*} \mathrm{p}<0.01$.

of neutrophils at $24 \mathrm{~h}$ (Table I; $\mathrm{p}<0.01)$. The increased percentage of neutrophils at $4 \mathrm{~h}$ and $24 \mathrm{~h}$ in the lavages of the treated groups (including saline), as compared to the untreated, is explained by the invasive technique used for administration to the lung (Table I; $p<0.01$ ). It should be noted that only macrophages and neutrophils were visible in BAL; no eosinophils were present.

With the exception of an infiltrate of lymphocytes that appeared around some bronchi after a priming or a boost dose of pOVA (Fig. 6c-d), no modifications to the lung structure, such as alterations to the trachea, bronchi or alveoli morphology, exudations, or infiltration of inflammatory cells, were observed $24 \mathrm{~h}$ following instillation of ovalbumin or pOVA in histology slides (Fig. 6).

\section{DISCUSSION}

In this article, we showed that antigen and DNA vaccines delivered to the lung of mice generated at least as high immunity systemically and locally in the lung as the i.m. injection of the same dose of vaccine, and that pulmonary vaccination did not result in pathological alterations to the lung tissue and in any apparent side effects in mice (Figs. 1, 4, and 6; Table I). These observations are in line with results previously collected with inhaled attenuated measles and inactivated influenza vaccines in preliminary clinical trials as well as with protection studies carried out in mice with Yersinia pestis F1 and V subunit vaccines co-encapsulated in polylactide microspheres (3-7). We extended these previous results to naked DNA vaccines and additionally supported the safety of vaccine delivery to the lung by direct analysis of the lung tissue.

Significant differences in the intensity and polarization of the immune response originated from the type and nature of the vaccine and smaller differences from the route of administration. The antigens induced serum and local (lung) IgG titers that were 1-2 logs higher than the plasmids (Fig. 1). However, pOVA tended to induce a stronger cellular response than the protein in proliferation assays (Fig. 4). Antigen and DNA immunization produced an immune response preferentially polarized toward the Th2 and Th1 type, respectively (Fig. 2), which is in agreement with respective modes of antigen presentation described in the literature (20). Compared to ovalbumin, HBsAg generated higher IgG2a and IgG2b titers relative to IgG1 titers (Fig. 2), suggesting increased differentiation of CD4 $\mathrm{T}$ cells into Th1 cells possibly due to the particulate nature and phospholipids content of HBsAg $(11,14,15)$. Depending on the type and nature of the vaccine, vaccine delivery to the lung produced an immune response that was greater or equal to the response generated in the quadriceps (Figs. 1 and 4). The characteristics of the immune response did not show any major differences with the route of administration, except that lymphocytes collected from lung-draining lymph nodes proliferated only after i.t. instillation of vaccines (Figs. 2 and 4). The limited differences that did arise in IgG titers and subclasses with the route of administration could originate from local differences in the type of dendritic cells, expression levels of the encoded antigen, and the impact of $\mathrm{CpG}$ motifs or other mechanisms (Figs. 1 and 2) (1,21,22).

Anti-ovalbumin and anti-HBsAg IgG but not $\operatorname{IgA}$ were found in lung washes after IT instillation of vaccines (Fig. $1 \mathrm{~b}$ and $\mathrm{d}$ ), indicating that the common mucosal immune system was probably not involved in the immune response. The predominance of specific $\operatorname{IgG}$ over $\operatorname{IgA}$ in BAL has previously been reported in cases of inhaled antigens and could be related to the poor development of the bronchus-associated lymphoid tissue in healthy mammals as well as to the preferential uptake of particulate material by these lymphoid ag- 
a

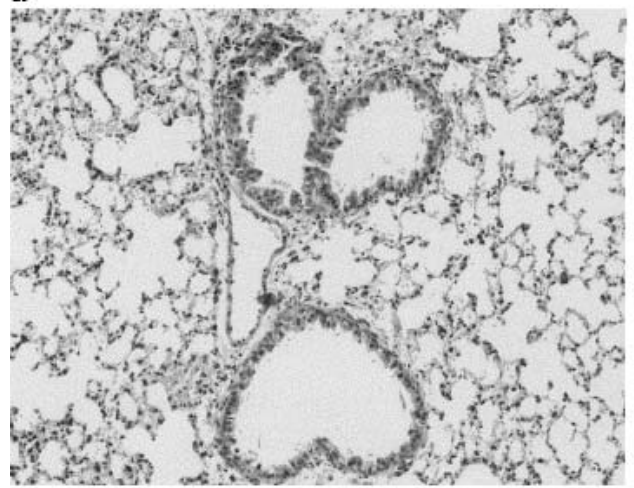

c

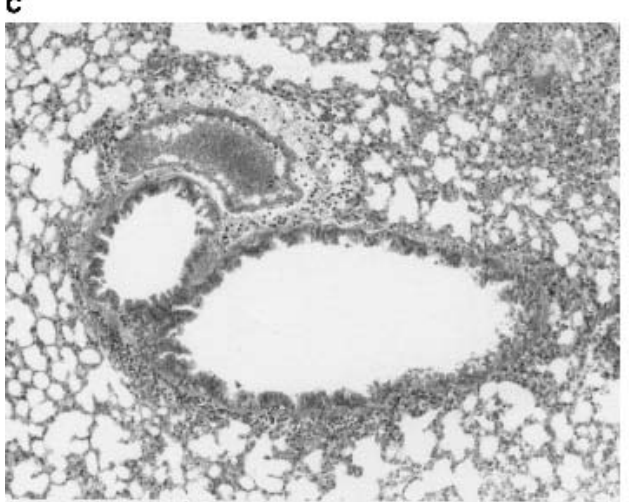

b

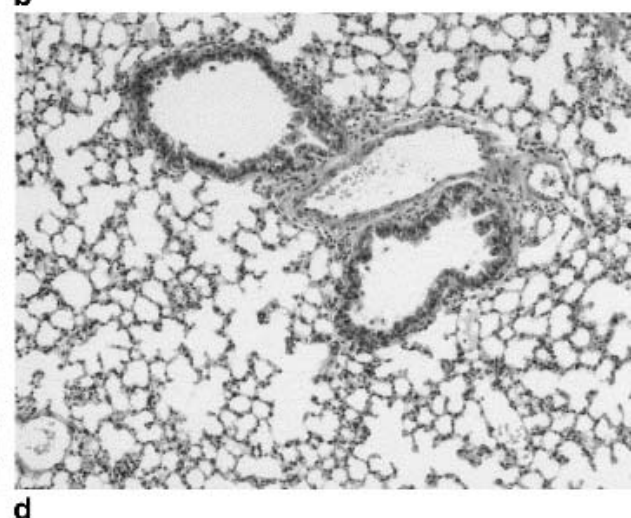

d

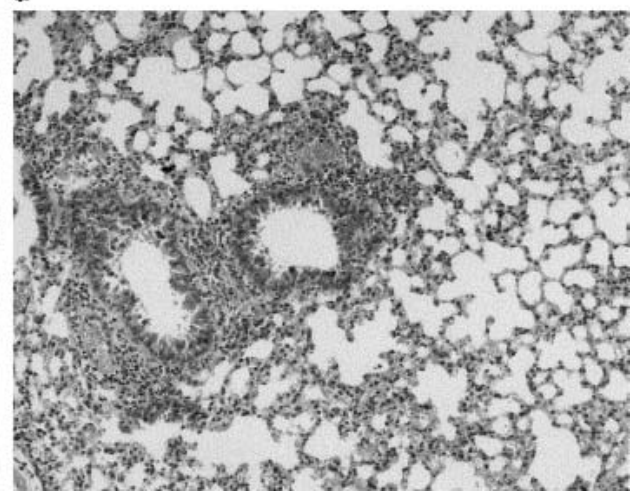

Fig. 6. Representative histological microphotographs of the lung $24 \mathrm{~h}$ following intrapulmonary immunization ( $\times 100$ ). (a) Saline or (b) ova was i.t. instilled on days 0 and 28 or pOVA was i.t. instilled (c) on day 0 or (d) on days 0 and 28 . The lung was analyzed (c) on day 1 or (a, b, d) day 29.

gregates $(6,18,21)$. In light of the correlation between $\mathrm{IgG}$ titers in BAL and serum (Fig. 3), it also remains to be determined if the reported improved protection of inhaled over injected vaccines in clinical trials resulted from actual enhanced defenses at the mucosa and/or from a global increase in immunity (3-5).

The equivalence of immunogenicity between a parenteral and a noninvasive route of administration appears unique to the respiratory tract and suggests a potential clinical application of aerosolized vaccines in respiratory as well as unrelated infections (1-7; Figs. 1 and 4). Delivering HBsAg or pHBsAg to the nasal mucosa did not induce any detectable specific immunity in our experiments. Other studies have shown that nasal or oral vaccines induced negligible immunity unless large doses and mucosal adjuvants, which are in general irritant, were used $(1,2)$. This situation likely results from the short time (minutes) of contact of the vaccine with the nasal or oral mucosa because of elimination by mucociliary clearance and/or local enzymes (23). This contrasts with the longer persistence (hours) of ovalbumin or other macromolecules in the airway lumen that was observed after i.t. instillation (Fig. 5) (24). It is to be noted that most acellular vaccines need adjuvants to develop protective immune responses by any route of administration, even injection. Although we demonstrated the equivalence of immunogenicity between the respiratory and muscle tissues at same doses without adjuvant, we have also shown that i.t. instillation of $\mathrm{HBsAg}$ resulted in an immune response lower than that of co-injection of HBsAg and alum, the marketed vaccine product (Fig. 1c). Therefore, the ultimate practical utility of aerosol vac- cines might require confirmation of efficacy and safety in humans, development of soft vaccine adjuvants for enhancing the immunity to the level of protection, and an appropriate aerosol formulation and inhaler device for delivery $(15,25)$. Successful stimulation of the common mucosal immune system in the airways is also needed to make the effectiveness of inhaled vaccines against respiratory pathogens superior to injected conventional formulations.

Few modifications to components in bronchoalveolar lavage fluid of the lung were induced by the repeated pulmonary delivery of ovalbumin and pOVA in mice (Table I). Although histological studies showed that the lung was not injured by the local administration of vaccines, an infiltration of lymphocytes around some bronchi appeared within $24 \mathrm{~h}$ after a priming or boost dose of pOVA (Fig. 6). This cellular infiltration is unlikely to result from an antigen-specific immune response because it requires a few days to develop, but might be related to the direct stimulatory effects of bacterial DNA on the immune system. Unmethylated $\mathrm{CpG}$ motifs have been shown to induce murine B cells to proliferate and secrete immunoglobulins in vitro and in vivo within a few hours of contact (22). The production of IL- 6 , IL-12, and IFN- $\gamma$ is also stimulated and creates a cytokine milieu that is probably involved in the polarization of the immune response (26).

Our experimental findings suggest that the immune response generated by the inhalation of vaccines is probably triggered at airway epithelial surfaces and that the specific immunity then develops systemically. Ovalbumin, a macromolecule of $45 \mathrm{kDa}$, penetrated the respiratory tissue poorly. It remained principally in the airway lumen for a few hours 
(Fig. 5) and was hardly transported in the systemic circulation following i.t. instillation. Because HBsAg is formed of 22-nm lipoprotein particles, it is likely that it diffused to an even lower extent than ovalbumin across the alveolar epithelium $(11,27)$. This supports a scenario whereby antigens delivered to the lung would be captured and processed over several hours by pulmonary dendritic cells, which would then be trafficked from the lung to regional lymph nodes and/or spleen and present the antigen to immune T cells (Fig. 4) (16,17). The IgG titers raised against ovalbumin or HBsAg in the lung correlated with their counterparts in serum following immunization by i.m. injection as well as by i.t. instillation (Fig. 3). This suggests that B cells would secrete antibodies in lymphoid tissues and that immunoglobulins would be transudated from the blood into the air space and/or that B cells producing specific antibodies penetrated the lung following both pulmonary and parenteral immunizations. In the case of pOVA, the dendritic cells transfected or having captured the antigen secreted by transfected cells would migrate to draining lymph nodes. Further understanding of the chain of immune events caused by inhaled vaccines will be sought through localization of pulmonary dendritic cells involved in the immunization, through the tracking of these cells up to the spleen, as well as through the identification and quantification of the immune cells penetrating the lung after pulmonary vaccination.

\section{ACKNOWLEDGMENTS}

We would like to thank K. Thielemans (Hematology and Oncology laboratory, Vrije Universiteit Brussel, Belgium), GlaxoSmithKline (Rixensart, Belgium), and Aldevron (Fargo, ND, USA) for donating the plasmid encoding ovalbumin, recombinant hepatitis $\mathrm{B}$ surface antigen (HBsAg), and the plasmid encoding $\mathrm{HBsAg}[\mathrm{pRc} / \mathrm{CMV}-\mathrm{HBs}(\mathrm{S})]$, respectively. Rita Vanbever is a Chercheur Qualifie of the Fonds National de la Recherche Scientifique (Belgium). This work was funded in part by UCL Special Funds for Research and the Actions de Recherches concertées, communauté française de Belgique. The English used in this manuscript was revised by Mr. Brendan McIntyre.

\section{REFERENCES}

1. J. Mestecky, Z. Moldoveanu, S. M. Michalek, C. D. Morrow, R. W. Compans, D. P. Schafer, and M. W. Russell. Current options for vaccine delivery systems by mucosal routes. J. Controlled Release 48:243-257 (1997).

2. R. Glueck. Review of intranasal influenza vaccine. Adv. Drug Deliv. Rev. 51:203-211 (2001).

3. A. B. Sabin. Immunization against measles by aerosol. Rev. Infect. Dis. 5:514-523 (1983).

4. A. Dilraj, F. T. Cutts, J. F. de Castro, J. G. Wheeler, D. Brown, C. Roth, H. M. Coovadia, and J. V. Bennett. Response to different measles vaccine strains given by aerosol and subcutaneous routes to schoolchildren: a randomized trial. Lancet 355:798-803 (2000).

5. R. H. Waldman, J. J. Mann, and P. A. Small, Jr. Immunization against influenza. Prevention of illness in man by aerosolized inactivated vaccine. JAMA 207:520-524 (1969).

6. J. E. Eyles, E. D. Williamson, I. D. Spiers, and H. O. Alpar. Protection studies following bronchopulmonary and intramuscular immunization with yersinia pestis $\mathrm{F} 1$ and $\mathrm{V}$ subunit vaccines coencapsulated in biodegradable microspheres: a comparison of efficacy. Vaccine 18:3266-3271 (2000).

7. A. Dilraj, F. T. Cutts, J. V. Bennett, J. F. de Castro, B. Cohen, and H. M. Coovadia. Persistence of measles antibody two years after revaccination by aerosol or subcutaneous routes. Pediatr. Infect. Dis. J. 19:1211-1213 (2000).
8. Y. L. Lai and H. Chou. Respiratory mechanics and maximal expiratory flow in the anesthetized mouse. J. Appl. Physiol. 88:939943 (2000).

9. J. S. Boyle, A. Silva, J. L. Brady, and A. M. Lew. DNA immunization: induction of higher avidity antibody and effect of route on T cell cytotoxicity. Proc. Natl. Acad. Sci. U. S. A. 94:14626-14631 (1997).

10. S. Wang, X. Liu, K. Fisher, J. G. Smith, F. Chen, T. W. Tobery, J. B. Ulmer, R. K. Evans, and M. J. Caulfield. Enhanced type I immune response to a hepatitis B DNA vaccine by formulation with calcium- or aluminum phosphate. Vaccine 18:1227-1235 (2000).

11. D. Diminsky, R. Schirmbeck, J. Reimann, and Y. Barenholz. Comparison between hepatitis B surface antigen (HBsAg) particles derived from mammalian cells $(\mathrm{CHO})$ and yeast cells (Hansenula polymorpha): composition, structure and immunogenicity. Vaccine 15:637-647 (1997).

12. M. E. Devey, K. Bleasdale, S. Lee, and S. Rath. Determination of the functional affinity of IgG1 and IgG4 antibodies to tetanus toxoid by isotype-specific solid-phase assays. J. Immunol. Methods 106:119-125 (1988).

13. R. F. Henderson. Biological markers in the respiratory tract. In R. O. McClellan and R. F. Henderson (eds), Concepts in Inhalation Toxicology. Taylor and Francis, Washington, DC, 1995, pp. $441-470$.

14. M. Isaka, Y. Yasuda, M. Mizokami, S. Kozuka, T. Taniguchi, K. Matano, J. Maeyama, K. Mizuno, K. Morokuma, K. Ohkuma, N. Goto, and K. Tochikubo. Mucosal immunization against hepatitis B virus by intranasal co-administration of recombinant hepatitis B surface antigen and recombinant cholera toxin B subunit as an adjuvant. Vaccine 19:1460-1466 (2001).

15. G. Gregoriadis, I. Gursel, M. Gursel, and B. McCormack. Liposomes as immunological adjuvants and vaccine carriers. J. Controlled Release 41:49-56 (1996).

16. W. Xia, C. E. Pinto, and R. L. Kradin. The antigen-presenting activities of Ia+ dendritic cells shift dynamically from lung to lymph node after an airway challenge with soluble antigen. J. Exp. Med. 181:1275-1283 (1995).

17. A. S. McWilliam, D. Nelson, J. A. Thomas, and P. G. Holt. Rapid dendritic cell recruitment is a hallmark of the acute inflammatory response at mucosal surfaces. J. Exp. Med. 179:1331-1336 (1994).

18. J. E. Eyles, I. D. Spiers, E. D. Williamson, and H. O. Alpar. Analysis of local and systemic immunological responses after intra-tracheal, intra-nasal and intra-muscular administration of microsphere co-encapsulated Yersinia pestis sub-unit vaccines. Vaccine 16:2000-2009 (1998).

19. C. LiCalsi, T. Christensen, J. V. Bennett, E. Phillips, and C. Witham. Dry powder inhalation as a potential delivery method for vaccines. Vaccine 17:1796-1803 (1999).

20. S. Gurunathan, D. M. Klinman, and R. A. Seder. DNA vaccines: immunology, application, and optimization. Annu. Rev. Immunol. 18:927-974 (2000)

21. J. Bienenstock, M. R. McDermott, and R. L. Clancy. Respiratory tract defenses: role of mucosal lymphoid tissues. In P. L. Ogra, J. Mestecky, M. E. Lamm, W. Strobber, J. Bienstock, and J. R. McGhee (eds), Mucosal Immunology. Academic Press, San Diego, 1999, pp. 283-292.

22. A. M. Krieg, A. K. Yi, S. Matson, T. J. Waldschmidt, G. A Bishop, R. Teasdale, G. A. Koretzky, and D. M. Klinman. Cpg motifs in bacterial-DNA trigger direct B-cell activation. Nature 374:546-549 (1995).

23. N. Jones. The nose and paranasal sinuses physiology and anatomy. Adv. Drug Deliv. Rev. 51:5-19 (2001).

24. R. H. Hastings, M. Grady, T. Sakuma, and M. A. Matthay. Clearance of different-sized proteins from the alveolar space in humans and rabbits. J. Appl. Physiol. 73:1310-1316 (1992).

25. D. A. Edwards. Delivery of biological agents by aerosols. Aiche J. 48:2-6 (2002)

26. D. M. Klinman, A. K. Yi, S. L. Beaucage, J. Conover, and A. M. Krieg. CpG motifs present in bacterial DNA rapidly induce lymphocytes to secrete interleukin 6, interleukin 12, and interferon gamma. Proc. Natl. Acad. Sci. U. S. A. 93:2879-2883 (1996).

27. D. A. Wall. Pulmonary absorption of peptides and proteins. Drug Deliv. 2:1-20 (1995). 